\title{
Typologie textuelle et connecteurs
}

\author{
Mohammed Jadir \\ Université Hassan II, Faculté des Lettres et Sciences Humaines, Mohammedia, Maroc \\ mohammedjadir@yahoo.fr
}

De nombreux travaux (Roulet et al. 1985, Schiffrin 1987, Hansen 1996, Moeschler 1996, Risselada 1998, et Jadir 2000a,b \& 2005a,b entre autres) ont montré que les particules connectives contribuent à la création de la cohérence du discours. De même, étant l'un des phénomènes linguistiques qui ne peuvent être étudiés adéquatement que dans une perspective textuelle, les connecteurs ont assuré l'extension de la Grammaire Fonctionnelle (GF) d'une grammaire de phrase en une Grammaire Fonctionnelle de Discours (GFD) (Hengeveld \& Mackenzie 2006 et 2008).

Toutefois, autant que nous sachions, peu de recherches en GF ont été réservées au rapport qu'entretient le type de discours avec les marqueurs de discours bien que certaines d'entre elles aient porté essentiellement sur les restrictions qu'impose le type de texte dans la sélection du type de Focus et de verbes de perception (Jadir 1995, 1998) et du type de modalité (Moutaouakil 1998) et d'illocution appropriées (Dik 1997a, b).

Dans cet article, notre objectif est d'examiner en quoi le type de discours codétermine dans une large mesure le choix des connecteurs. Ainsi, après un bref rappel du cadre théorique dans lequel s'inscrira notre travail (sect.1), nous établirons une comparaison entre la dichotomie benvenistienne Récit/Discours et la dichotomie genevoise monologal/dialogal (sect. 2 et 3) pour montrer que le type de texte a un impact sur les formes verbales et personnelles, sur la deixis, sur les verbes modaux et sur les verbes performatifs. Dans la section 4, nous procèderons à une statistique de la fréquence des différents types de particules (e.g. causales, adversatives, consécutives) dans un corpus diversifié : L'Assommoir d'Emile Zola (Roman), Les Liaisons dangereuses de Choderlos de Laclos (Roman épistolaire) et Les Fausses confidences de Marivaux (Pièce de théâtre). La dernière section sera consacrée à une explication fonctionnelle de dépouillements obtenus et une analyse contrastive des différents types de particules.

\section{Vers une grammaire fonctionnelle du discours}

Dans cette section, il sera question de quelques concepts-clés de la Théorie de la GF.

\subsection{Trois Perspectives}

Les exigences des adéquations (pragmatique, psychologique et typologique) imposées à la GF permettent de concevoir cette théorie comme un module dans le modèle intégré de l'Utilisateur du Langage Naturel (ULN). La GF, munie des autres modules (i.e. épistémique, logique, perceptuel et social), devrait nous permettre de comprendre comment les ULN sont capables de communiquer les uns avec les autres à travers l'interaction verbale.

Dik (1997b: 409) note que les ULN ne communiquent pas en termes de clauses (= phrases) isolées, mais en termes de passages plus longs et plus complexes auxquels il convient de conférer le terme de 'discours'. Celui-ci n'équivaut pas à une simple « séquence arbitraire de clauses ». Pour Dik, une théorie 'optimale' de la clause s'avère impossible puisqu'elle est conçue comme faisant partie de la théorie plus large de la compétence communicative de l'ULN et que la structure interne de toute clause est sensible à de nombreux facteurs d'ordre discursif.

Dik (1997b: ch. 18) esquisse une théorie fonctionnelle du discours qui a pour but de rendre compte des problèmes relatifs à la production, l'organisation et l'interprétation du discours. L'auteur propose d'approcher ces problèmes à partir de trois perspectives distinctes mais complémentaires: les décisions globales du discours (1.1.1), les structures globales du discours (1.1.2) et la cohérence du discours (1.1.3). 


\subsubsection{Les décisions globales du discours}

Lorsqu'un L(ocuteur) bâtit un discours, il prend plusieurs décisions qui affectent l'ensemble de clauses qui constituent le discours. A cet égard, de nombreuses décisions globales du discours sont à distinguer. La première décision concerne le désir de L d'entamer (ou non) un événement de discours. Selon Hymes (1972), un événement de discours est défini comme un événement social et interpersonnel gouverné par des paramètres tels que les participants ( $\mathrm{L}$ et $\mathrm{A}($ (llocutaire)), leurs droits et devoirs vis-à-vis de l'interaction et du contenu, le temps, le lieu et la situation d'énonciation.

Après cette étape, L doit choisir un genre/type de discours et un style approprié à son discours. Les décisions temporelles et les illocutions discursives ne sont pas à négliger. Les types de discours sont classifiés selon des facteurs tels que: le Moyen (oral ou écrit), la Participation (monologue, dialogue, polylogue), la Relation des participants (face-à-face direct, semi-direct (e.g. à travers le téléphone, la radio, la télévision) ou indirect (e.g. à travers un texte écrit)), la Formalité (degré d'institutionnalisation de l'événement du discours, degré de formalité du style de l'interaction) et le But communicatif (narratif, argumentatif, didactique, etc.).

Les exemples de genre au sens que ce terme prend ici sont la Conversation, l'Interview, l'Exposé, l'Appel téléphonique, le Sonnet, la Réunion, la Lettre et le Conte de fées. Dik note que le choix d'un genre a d'importantes implications sur la construction linguistique d'un discours aussi bien au niveau global (au niveau de la macrostructure) qu'au niveau local (au niveau de la microstructure).

\subsubsection{Les structures globales du discours}

Tout discours a une structure hiérarchique et stratifiée une fois considéré comme un produit fini. Cette structure peut être assimilée à la structure de la clause en ce qui concerne, au moins, deux types de complexité: la stratification et la récursion. Parallèlement aux strates fonctionnelles distinguées au niveau de la clause, Dik (1997b : 245) établit une stratification qui comprend (a) un niveau interpersonnel ayant trait à l'interaction entre $\mathrm{L}$ et $\mathrm{A}$ et à leur évaluation/attitude (émotionnelle ou critique) vis-à-vis du discours, et (b) un niveau représentationnel ayant trait à la façon dont le contenu du discours est organisé et aux éléments discursifs (i.e. Participants, Faits et Etats de Choses (EdC)) qui ont assuré la transmission de ce contenu. Le second type de complexité dans le discours est dû à la récursion. Selon Dik «la récursion est impliquée dans toute situation dans laquelle une unité du type $\mathrm{U}$ peut contenir une sousunité du même type U, tel que, dans U, l'organisation typique de U peut se reproduire » (1997b: 427).

Après avoir examiné la structure du discours d'un point de vue structurel, Dik aborde cette structure d'un point de vue fonctionnel. L'auteur suppose que toutes les relations intraclausales noyau-satellite peuvent être projetées sur le niveau interclausal. Il donne les exemples suivants:

(1) Le niveau interpersonnel

a. $U_{1}$ motive $U_{2}$

b. $\mathrm{U}_{1}$ fournit un arrière-plan pour $\mathrm{U}_{2}$

Le niveau représentationnel

a. $U_{1}$ développe $U_{2}$

b. $U_{1}$ spécifie une condition pour $\mathrm{U}_{2}$

\subsubsection{La cohérence du discours}

«Quand un discours est proprement construit, il devient cohérent en ce sens que les différentes parties qui le constituent se suivront d'une façon naturelle et interprétable. Ceci vaut aussi bien pour la succession 
locale des clauses (la cohérence locale) que pour la succession globale des unités du discours (la cohérence globale) » (Dik 1997b : 433). Plusieurs facteurs contribuent à la cohérence du discours: le 'Cadre' (Frame), i.e. la connaissance organisée concernant ce qui peut être fait et dit dans une situation institutionnelle donnée, le 'lien Queue-tête' (Tail-head linking) où une clause commence par un constituant qui résume brièvement une partie cruciale de la clause ou du contexte précédent (De Vries 1989), l'iconicité temporelle, la continuité topicale, la focalité ${ }^{1}$ et les connecteurs qui figurent dans des schémas ayant la forme suivante :

(2) Clause(s) Précédente(s). Connecteur, Clause Nouvelle.

où le rôle du connecteur consiste en premier lieu à lier la clause nouvelle à la (aux) clause(s) précédente(s) tout en spécifiant une relation d'ordre sémantico-pragmatique entre elles.

\subsection{Deux directions}

La structure sous-jacente de la clause (SSC) telle qu'elle est conçue en GF (Dik 1989, 1997a) consiste en cinq strates appelées 'prédication nucléaire', 'prédication centrale', 'prédication étendue', 'proposition' et 'illocution'. La conversion d'une strate en une autre qui lui est supérieure s'effectue par l'adjonction de deux types de moyens: des moyens grammaticaux (les opérateurs $(\pi)$ ) et des moyens lexicaux (les satellites $(\sigma))^{2}$. Ces strates, qui sont supposées être pertinentes pour l'analyse des langues naturelles, désignent plusieurs types d'entités qui sont listés dans le Tableau suivant (Dik 1989 : 50, 1997a : 93) :

Tableau 1. Structure, type d'entité, ordre et variable

\begin{tabular}{lccc}
\hline \multicolumn{1}{c}{ Unité structurale } & Type d'entité & Ordre & Variable \\
\hline Clause & acte de langage & 4 & $\mathrm{E}_{\mathrm{i}}$ \\
Proposition & fait possible & 3 & $\mathrm{X}_{\mathrm{i}}$ \\
Prédication & état de choses & 2 & $\mathrm{e}_{\mathrm{i}}$ \\
Terme & entité & 1 & $\mathrm{x}_{\mathrm{i}}$ \\
Prédicat & propriété/relation & & $\mathrm{f}_{\mathrm{i}}$ \\
\hline
\end{tabular}

Le développement du modèle actuel de la phrase (i.e. la GF) en un modèle de discours fait l'objet d'un grand débat. Toutefois, il y a deux directions particulièrement intéressantes pour l'étendre : la première, appelée "stratification expansionniste" (upward layering), consiste à pourvoir la SSC d'une strate supérieure, soit la strate textuelle (cf. Hengeveld 1997; Cuvalay 1997; Moutaouakil 1998; Jadir 1998, etc.), la seconde interprète la variable E (Dik 1989) comme un produit d'acte de langage, i.e. comme un énoncé (utterance) (cf. Kroon 1997; Bolkestein 1998; Vet 1998). Ces auteurs proposent de distinguer deux modules séparés : un module grammatical et un module pragmatique dans lequel les aspects pragmatiques du langage devraient être pris en charge ${ }^{3}$.

\section{Dichotomie Récit-Discours}

Pour une description adéquate du système verbal et du système des pronoms en français, Benveniste (1966: 237-266) distingue deux plans d'énonciation correspondant à deux types de discours: le Discours et le Récit.

Le Discours, pris dans sa plus large extension, désigne « toute énonciation supposant un locuteur et un auditeur, et chez le premier l'intention d'influencer l'autre en quelque manière » (p. 242). Le Discours est marqué alors subjectivement dans la mesure où il implique un sujet d'énonciation (i.e. un locuteur) qui s'adresse à un récepteur, c'est le cas, par exemple, des correspondances, des mémoires, d'ouvrages didactiques et du dialogue.

Par opposition au Discours, le Récit se caractérise par la présentation objective des événements survenus dans le passé. Autrement dit, ce type de discours exclut toute référence à l'auteur et proscrit tout ce qui est 
étranger au récit des événements, i.e. à l'histoire (e.g. interventions, réflexions, commentaires, comparaisons). «A vrai dire, il n'y a même plus alors de narrateur. Les événements sont posés comme ils se sont produits à mesure qu'ils apparaissent à l'horizon de l'histoire. Personne ne parle ici ; les événements semblent se raconter eux-mêmes » (Benveniste 1966: 241). Relèvent de ce type de texte les traités scientifiques, les textes historiques, les narrations, etc.

Le premier argument avancé en faveur de l'opposition Récit-Discours consiste en les formes verbales et les pronoms personnels. En effet, le registre des temps verbaux est bien plus large dans le Discours que dans le Récit du moment que tous les temps (i.e. le présent, le futur (simple et composé) et le parfait) sont possibles excepté l'aoriste (i.e. le passé simple). Cette forme verbale, bannie du Discours, constitue la forme typique du Récit. Communs aux deux types de discours sont l'imparfait et le plus-que-parfait.

Concernant les formes personnelles du verbe, le Discours emploie la première (je), la deuxième $(t u)$ et la troisième $(i l)$ forme, tandis que dans le Récit, seule la non-personne (i.e. $i l$ ) est admise. Il ressort alors qu'à l'encontre du Discours qui exclut l'aoriste, le Récit qui l'utilise ne retient que la forme qui lui est convenable, en l'occurrence la troisième personne. Considérons, à titre d'illustration, le Récit narratif (3) extrait de Germinal p. 20 :

(3) Du reste, Catherine fut prête la première. Elle enfila sa culotte de mineur, passa la veste de toile, noua le béguin bleu autour de son chignon ...

Dans ce Récit historique, le temps utilisé par excellence pour la description des faits est l'aoriste associé avec la forme (non) personnelle elle. Signalons que toute tentative de substitution d'un temps parfait (e.g. le passé composé) à l'aoriste ou des formes je/tu à la forme elle sera vouée à l'échec dans la mesure où elle donnera lieu à des textes bizarres. En témoignent, respectivement, les segments (4) et (5) ${ }^{4}$ :

(4) ?? Du reste, Catherine a été prête la première. Elle a enfilé sa culotte de mineur, a passé la veste, a noué le béguin bleu autour de son chignon...

(5) ?? Du reste, je fus prêt le premier. J'enfilai ma culotte de mineur, passai la veste de toile, nouai le béguin bleu autour de mon chignon...

Par ailleurs, la dichotomie Récit/Discours est corroborée par un deuxième argument consistant en les expressions déictiques, les verbes performatifs et les verbes modaux.

Le Discours se distingue nettement du Récit par son recours à une classe 'd'indicateurs' qui réfèrent à l'acte de langage performé par le locuteur, i.e. à 'l'instance de discours'. Relèvent de cette série d'indicateurs déictiques (a) les démonstratifs (e.g. ce) qui servent à désigner un objet par ostension simultanée à la présente instance de discours, (b) les adverbes ici et maintenant qui renvoient à l'instance spatio-temporelle de l'énonciation, et (c) les expressions adverbiales du type de aujourd'hui, hier, demain, etc. qui délimitent le même type de relation entre le temps et l'instance de discours (je/tu).

Il est à noter que parallèlement à cette classe d'unités linguistiques qui spécifient le rapport de l'indicateur à l'instance de discours, la langue fournit une autre classe correspondante, propre au Récit, référant aux objets, aux lieux et aux temps de l'histoire. Aussi avons-nous les corrélations 'terme à terme' telles que: jelil, icillà, maintenant/alors, hier/la veille, demain/le lendemain, etc.

Les verbes subjectivement modalisés (e.g. croire, présumer, supposer, conclure...) et les verbes performatifs (e.g. jurer, promettre, garantir, certifier...) ont pour trait commun le fait de se comporter différemment selon qu'ils sont assumés par une personne (je) ou une non-personne (il). Pour illustrer les effets de sens que produit le changement de personnes dans ces deux types de verbes, considérons les exemples suivants :

a. Je présume que Jean a reçu la lettre.

b. Il présume que Jean a reçu la lettre.

a. Je jure que Paul est innocent. 


\section{b. Il jure que Paul est innocent.}

En (6a) je présume caractérise l'attitude du locuteur vis-à-vis du contenu propositionnel de l'énoncé qui suit. En (6b), par contre, le verbe présumer précédé d'une troisième personne (il) décrit une opération. De même, en (7a), en proférant je jure, le locuteur accomplit l'acte de serment ; il s'agit, en d'autres termes, d'un engagement de sa part. Le changement de personne illje comme en (7b) convertit l'accomplissement de l'acte en une simple description.

Les phrases (6a) et (7a) comportent, chacune, une seule et véritable proposition précédée d'un indicateur de subjectivité, en l'occurrence le verbe d'attitude propositionnelle je présume et le verbe performatif je jure respectivement. Les phrases (6a) et (7a) sont alors des phrases simples, contrairement à leurs contreparties (6b) et (7b) qui sont des phrases complexes puisqu'elles consistent en un verbe matriciel et un complément propositionnel.

Il en découle que les verbes performatifs et les verbes véhiculant une modalité subjective sont le propre du Discours parce qu'ils réfèrent au locuteur.

Nous pouvons, au terme de cette présentation, schématiser les différents moyens linguistiques, avancés supra en faveur de l'opposition Récit/Discours sous forme du Tableau récapitulatif suivant :

Tableau 2. Moyens linguistiques et typologie Récit/Discours

\begin{tabular}{|c|c|c|c|c|c|}
\hline \multirow{2}{*}{$\begin{array}{c}\text { Type de } \\
\text { Discours }\end{array}$} & \multicolumn{2}{|c|}{ Formes } & \multirow{2}{*}{ Deixis } & \multicolumn{2}{|c|}{ Verbes } \\
\cline { 2 - 3 } \cline { 5 - 5 } & verbales & personnelles & & modaux & performatifs \\
\hline Récit & Aoriste & $3 \mathrm{e}$ & - & $\varnothing$ \\
\hline Discours & $\begin{array}{c}\text { Présent, Futur, } \\
\text { Parfait }\end{array}$ & $1^{\mathrm{re}}, 2 \mathrm{e}, 3 \mathrm{e}$ & + & + \\
\hline $\begin{array}{c}\text { Récit \& } \\
\text { Discours }\end{array}$ & Imparfait, PQP & $3 \mathrm{e}$ & $\varnothing$ & $\varnothing$ \\
\hline
\end{tabular}

\section{Dichotomie monologal-dialogal}

Roulet et al. $(1985$ : ch.1) et Kroon $(1994,1995)$ considèrent que le type de discours réfère au mode communicatif associé avec un texte tel que, par exemple, le monologue, le dialogue et le polylogue. Bien plus, le type de discours est doté d'un aspect formel et d'un aspect fonctionnel.

Au niveau formel, un discours peut être dialogal ou monologal. Le discours monologal est produit par un seul locuteur/scripteur, alors qu'un discours dialogal est produit par au moins deux locuteurs/scripteurs. Ces deux types de structures présentent, bien entendu, des structures différentes.

Au niveau fonctionnel, la distinction s'effectue entre un discours monologique à structure d'intervention, dont les constituants immédiats sont liés par des fonctions interactives, et un discours dialogique à structure d'échange, dont les constituants immédiats sont liés par des fonctions illocutoires initiative et réactive.

En termes de Kroon, un texte dialogique comporte des mouvements alternatifs des participants au discours qui, dans leur totalité, constituent un échange interactionnel. Un texte monologique, quant à lui, ne consiste pas en des mouvements initiatif et réactif, mais consiste seulement en un mouvement unique.

La combinaison des deux paramètres forme et fonction donne lieu au quatre types de discours visualisés dans le Tableau suivant: 
Tableau 3. Forme \& fonction

\begin{tabular}{ll} 
FORME & FONCTION \\
\hline 1. dialogal & dialogique \\
2. dialogal & monologique \\
3. monologal & dialogique \\
4. monologal & monologique \\
\hline
\end{tabular}

Les types de discours dialogal dialogique et monologal monologique sont les types les plus aisément repérables dans la mesure où ils réfèrent aux monologues et aux dialogues dans le sens le plus conventionnel du terme.

Etant donné que la distinction monologique-dialogique peut être conçue comme recouvrant la distinction entre discours faisant intervenir un énonciateur principal et discours faisant intervenir au moins deux énonciateurs principaux, le monologal dialogique est alors un texte produit par un locuteur/scripteur mais qui donne la parole à deux énonciateurs principaux. Un roman épistolaire, par exemple, est monologal dans la mesure où il est produit par un seul locuteur (i.e. l'auteur), et dialogique dans la mesure où il simule des échanges, formés d'interventions (épistolaires), entre les personnages.

Le dernier type de discours, i.e. le discours dialogal monologique pose, semble-t-il, un problème puisqu'il devrait être produit par au moins deux locuteurs/scripteurs et avoir une structure d'intervention.

Quoique peu fréquent, ce type de discours existe; il se réalise chaque fois que le discours de l'énonciateur principal comporte des « voix enchâssées », i.e. chaque fois qu'une intervention constitutive d'échange comprend des échanges enchâssés.

Outre les paramètres formel/fonctionnel, la double opposition entre, d'une part, la présence d'un ou deux locuteurs/scripteurs, et, d'autre part, la présence d'un ou deux énonciateurs principaux constitue un paramètre supplémentaire pour mieux définir les quatre types de discours, comme il ressort du Tableau 4:

Tableau 4. Locuteur/énonciateur et type de discours

\begin{tabular}{|c|c|c|}
\hline & un locuteur/scripteur & deux locuteurs/scripteurs \\
\hline $\begin{array}{c}\text { un énonciateur principal } \\
\text { (structure d'intervention) }\end{array}$ & $\begin{array}{c}\text { MONOLOGAL } \\
\text { MONOLOGIQUE }\end{array}$ & $\begin{array}{c}\text { DIALOGAL } \\
\text { MONOLOGIQUE }\end{array}$ \\
\hline $\begin{array}{c}\text { deux énonciateurs } \\
\text { principaux (structure } \\
\text { d'échange) }\end{array}$ & MONOLOGAL & DIALOGAL \\
\hline
\end{tabular}

S'inspirant de Bakhtine (1970,1978), de Ducrot et al. (1980) et de Ducrot (1982, 1983), Roulet et al. (1985) s'intéressent, dans leur modèle de la structure hiérarchique du discours, à la pluralité des voix dans l'intervention, i.e. dans le discours monologique. Aussi distinguent-ils entre trois types de structures monologiques: la structure monologique monophonique, la structure monologique polyphonique et la structure monologique diaphonique.

On a affaire à un discours monologique monophonique quand l'intervention fait entendre une seule voix, un seul auteur qui s'identifie avec l'énonciateur. Cependant, lorsqu'une intervention fait entendre deux voix imputables à deux auteurs différents (i.e. l'énonciateur et quelqu'un d'autre), il s'agit, dans ce cas, d'une structure monologique polyphonique. Enfin, le discours monologique diaphonique est impliqué chaque fois qu'une intervention fait entendre deux voix : celle de l'énonciateur et celle du destinataire ${ }^{5}$.

Il est possible de reproduire graphiquement les Tableaux (3) et (4) comme dans le Graphique 1:

Graphique 1. Types de discours 


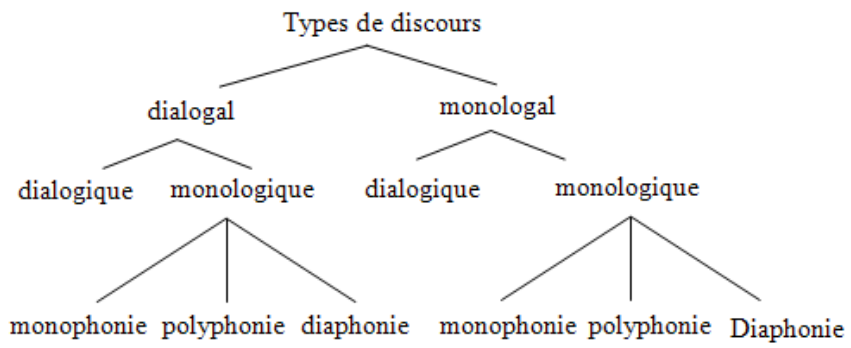

\section{Remarques}

L'examen des quatre types de discours compris dans le Graphique 1, permettrait, a priori, de conclure qu'aucun d'entre ces types ne correspond parfaitement au Récit au sens benvenistien du terme. Aussi estil possible même d'avancer que ces types dans leur totalité sont inclus dans le Discours qui, lui, peut comprendre des discours à structure aussi bien dialogale que monologale.

En effet, le discours dialogique (qu'il soit dialogal ou monologal) implique (ou simule) une structure d'échange avec deux protagonistes, i.e. deux énonciateurs. Il s'agit alors d'un sous-type de Discours dit 'Discours conversationnel' (Dc). La structure de l'échange interactionnel se manifeste, clairement, surtout dans le cas des discours dialogaux qui comportent deux locuteurs/scripteurs.

Pour ce qui est du discours monologique ((poly) et (dia)phonique) dialogal et monologal, il a une structure d'intervention constitutive d'échange et implique des 'voix enchâssées' ce qui l'éloigne du Récit qui proscrit tout ce qui est étranger à l'histoire.

Enfin, le discours monologique (monologal) monophonique présente, certes, une certaine affinité avec le Récit dans la mesure où il ne fait entendre que la seule voix de l'énonciateur qui le prend en charge. Cependant, ce type de discours n'est pas sans s'apparenter avec le Discours. Soit les exemples suivants :

$$
\begin{aligned}
& \text { C: - hm-non parce que c'est juste pour un conte (...) } \\
& \text { C: j'trouve ça m'embête un peu comme ça parce que j'avais pris cet été un vol aller simple } \\
& \text { c'était } 198 \text { que j'avais payé - }
\end{aligned}
$$

Dans ces exemples, empruntés à Roulet et al. (1985: 72), les auteurs des deux actes constitutifs de l'intervention dans les deux cas sont identiques et s'identifient avec l'énonciateur principal. En revanche, les interventions en question sont extraites de deux conversations analysées par Roulet et al. dans (1.3.2) et font référence à bon nombre de traits linguistiques qui caractérisent le Discours, e.g. l'implication du sujet d'énonciation ( $\mathrm{je}$ ), le temps verbal (le présent), l'emploi de la première forme personnelle (je), l'expression déictique (cet été), etc.

Ceci dit, nous pensons qu'une typologie de discours telle que celle donnée dans le Graphique 1 qui se veut englobant, devrait être enrichie, du moins, par l'adjonction d'un autre type correspondant au Récit.

Dans ce travail, nous continuons à adopter la dichotomie Récit/Discours vu son adéquation tout en référant, en cas de besoin, à la typologie donnée supra et à celle de Dik (1997b) (cf. 1.1.1).

\section{$5 \quad$ Impacts du type de discours}

Dans cette section, nous procèderons à des statistiques des différents types de particules connectives relevées dans le corpus objet de notre investigation et à une explication fonctionnelle des résultats de notre dépouillement. 


\subsection{Dépouillements}

Pour pouvoir tester l'impact du type de discours sur le choix des connecteurs, nous avons procédé à une statistique de la fréquence des particules causales (i.e. car, parce que, puisque, en effet, même et d'ailleurs), des particules adversatives (i.e. bien que, pourtant, néanmoins, cependant, quand même, mais et tandis que), des particules consécutives (i.e. ainsi, aussi, par conséquent, donc et alors) et de l'interjectif eh bien dans les données tirées de L'Assommoir (ASS) d'Emile Zola (Roman), Les Liaisons dangereuses (LD) de Choderlos de Laclos (Roman épistolaire) et les Fausses confidences (FC) de Marivaux (Pièce de théâtre).

Il est à noter que la notion de connecteur, de particule connective ou de marqueur de discours, que nous employons dans ce travail de façon interchangeable, est pris ici dans son sens large comprenant conjonctions, adverbes, etc. (cf. (Roulet et al. 1985, Schiffrin 1987, Stubbs 1983) et servant à articuler deux actes de discours (Kroon 1997, Hengeveld \& Mackenzie 2006 et 2008). La notion d'acte, définie comme la plus petite unité du discours, peut se réaliser sous forme d'un terme, d'une clause, etc. Les particules peuvent connecter également des mouvements ou des paragraphes.

Les résultats de notre dépouillement sont visualisés dans le Tableau suivant:

Tableau 5. Fréquence des connecteurs

\begin{tabular}{|l|c|c|c|}
\hline \multicolumn{1}{|c|}{ Connecteurs } & \% dans FC & \% dans LD & \% dans ASS \\
\hline Car & 8.74 & 10.47 & 2.71 \\
Parce que & 1.46 & 2.74 & 5.41 \\
Puisque & 2.92 & 2.02 & $/$ \\
En effet & 1.46 & 4.74 & 1.16 \\
Même & 0.48 & $/$ & 0.77 \\
D'ailleurs & 0.48 & $/$ & 0.38 \\
Bien que & $/$ & 0.12 & 0.38 \\
Pourtant & 6.79 & 7.36 & 10.05 \\
Néanmoins & $/$ & $/$ & $/$ \\
Cependant & 0.97 & 6.35 & 14.50 \\
Quand même & $/$ & $/$ & $/$ \\
Mais & 39.80 & 45.01 & 36.75 \\
Tandis que & $/$ & 1.74 & 7.54 \\
Ainsi & 1.46 & 1.38 & $/$ \\
Aussi & $/$ & 1.99 & 0.96 \\
Par conséquent & 0.48 & 0.12 & $/$ \\
Donc & 23.30 & 11.34 & 19.39 \\
Alors & $/$ & 3.24 & $/$ \\
Eh bien & 11.66 & 0.12 & \\
\hline
\end{tabular}

Les données puisées dans L'Assommoir et qui ont constitué le champ de notre investigation peuvent être conçues comme un «spécimen de récit» selon l'expression de Benveniste (1966: 239), puisque nous n'avons tenu compte dans notre calcul que des occurrences des connecteurs articulant deux segments narratifs (ou un segment narratif et un autre non-narratif, ce qui marque « une intrusion » du Discours dans le Récit). La désignation ASS dans ce travail renvoie exclusivement aux données étudiées. Au reste, s'il est possible, bien entendu, de considérer le corpus constitutif des FC comme un texte-Discours (conversationnel) du moment qu'il s'agit d'une instanciation de discours dialogal dialogique, il n'est pas possible, semble-t-il, de trancher aisément dans le cas des $\mathrm{LD}^{6}$.

En effet, le texte des LD, comme tout roman épistolaire, présente des affinités aussi bien avec le premier type qu'avec le second type. Etant monologal, le texte des LD s'apparente avec celui de l'ASS puisque, 
dans les deux cas, la totalité de l'histoire est prise en charge par une seule entité, i.e. l'auteur. Et, étant dialogique, les LD s'assimile, avec les FC puisqu'il simule des échanges conversationnels: dans les FC l'auteur attribue les dialogues aux protagonistes (personnages), de même, chaque lettre peut-elle être prise comme l'équivalent d'une réplique dans un dialogue.

Cette mise au point semble pertinente dans la mesure où le caractère hybride des lettres (LD) se traduit au niveau des résultats numériques et, corollairement, corrobore le constat de l'impact du type de discours sur la sélection des marqueurs de discours.

Les marqueurs donc et eh bien qui sont propres au Discours conversationnel $(23.30 \%$ vs $11.66 \%$ respectivement dans les FC) sont présents également dans les LD (11.34\% vs $0.12 \%$ respectivement) alors qu'ils sont complètement absents (/) dans l'ASS.

Inversement, les connecteurs alors et tandis que qui sont exclusifs au Récit narratif (i.e. l'ASS) $(19.39 \%$ vs $7.54 \%$ ) sont assez fréquents dans les LD (3.24\% vs $1.74 \%$ ) mais les FC en est totalement dépourvu (/).

Par ailleurs, la similitude de l'impact du type de discours que manifeste les LD avec les FC est illustrée également par la haute fréquence du connecteur car $(10.47 \%$ vs $8.74 \%)$ par opposition à l'ASS où ce connecteur manifeste une fréquence très basse $(2.71 \%)$. Les indices chiffrés concernant le concessif cependant corroborent, d'autre part, la ressemblance des types de discours dans l'ASS (14.50\%) et les LD (6.35\%) par opposition aux FC (0.97\%).

Il est communément admis que le Récit narratif (e.g. l'ASS) et le Discours conversationnel (e.g. FC) diffèrent nettement. Cette différence se reflète au niveau de la fréquence des connecteurs. Autrement dit, chaque type de discours sélectionne le type de connecteurs qui lui est approprié.

A titre d'illustration, la consécution est quasi strictement exprimée dans le Récit narratif par alors ( $19.39 \%$ de $20.35 \%$ que représente l'ensemble des particules comptées) alors que c'est le marqueur donc qui prédomine dans le cas du Discours conversationnel (23.30\% de 25.24\%). Notons que les liens aussi et ainsi en dépit de leur fréquence très basse sont en 'distribution complémentaire'; le premier est présent seulement dans le Récit narratif ( $0.96 \%)$, le second ne l'est que dans le Discours conversationnel (1.46\%).

Concernant les connecteurs 'contre-argumentatifs' en termes de Roulet et al. (1985), ils sont plus fréquents dans le Récit narratif (69.22\%) que dans le Discours conversationnel (47.56\%). En effet, excepté le concessif pourtant (quoique plus fréquent dans l'ASS (10.05\%) que dans les FC (6.79\%)) et l'oppositif mais, point commun des deux types de discours ( $36.75 \%$ vs $39.80 \%$ ), le Récit narratif recourt à d'autres marqueurs adversatifs, e.g. cependant et tandis que qui sont, respectivement, soit d'une fréquence très faible dans le Discours conversationnel $(14.50 \%$ vs $0.97 \%)$ ou totalement absents $(7.54 \%$ vs / ) de ce type de texte.

La fréquence d'occurrence des connecteurs même et d'ailleurs est très basse aussi bien dans le Récit narratif $(0.77 \%$ vs $0.38 \%)$ que dans le Discours conversationnel ( $0.48 \%$ vs $0.48 \%)$. Le même constat vaut également pour en effet (1.16\% vs 1.46\%). Les autres marqueurs de cause car et parce que se comportent, toutes proportions gardées, de la même manière que les consécutifs aussi et ainsi; le premier est plus fréquent dans le Discours conversationnel (8.74\%) comparativement au Récit narratif (2.71\%), le second, inversement, est moins fréquent dans le Discours conversationnel (1.46\%) relativement au Récit narratif $(5.41 \%)$.

Enfin, le connecteur eh bien ne peut figurer que dans le Discours conversationnel, le Récit narratif bloque l'occurrence de ce type de particule.'

Ce faisant, comment expliquer fonctionnellement la distribution des particules connectives en fonction du type de discours; autrement dit, quelle explication fonctionnelle peut-on fournir à l'impact du type de discours sur la sélection des connecteurs? 


\subsection{Vers une explication fonctionnelle}

Le caractère polyphonique et interactionnel des marqueurs de discours eh bien ${ }^{8}$ et puisque fait, par exemple, que ceux-ci n'apparaissent que dans le Discours conversationnel. En d'autres termes, dans un Récit (narratif), qui exclut toute référence aux participants, on ne s'attendrait pas à rencontrer des connecteurs dont la fonction primaire consiste à désigner l'acte d'énonciation. Ce type de connecteurs qui relèvent, en termes de GF, du niveau illocutionnaire nécessite la présence d'un locuteur et sert à spécifier la manière dont celui-ci désire que l'acte de langage soit compris par l'allocutaire.

Pour montrer la spécificité du marqueur puisque, nous l'avons comparé à car (cf. Jadir 2005b, 2006). Parmi les arguments avancés dans ce sens, nous nous limitons aux deux suivants :

(i) Les particules connectives car et puisque supposent, certes, toutes les deux, deux actes d'énonciation distincts, dont le deuxièmes constitue une motivation du premier. Mais elles diffèrent eu égard au 'locuteur' auquel est imputée la responsabilité de cette motivation. Ainsi, alors que les deux clauses cordonnées au moyen de car sont prises en charge par le même locuteur, le locuteur de la clause principale, dans le cas de puisque, se différencie de 'l'énonciateur' ou du 'Locuteur original' (en termes de Dik 1997b), de la clause subordonnée, il n'en est que le 'Rapporteur'. L'agrammaticalité douteuse de (9b), dans ce contexte, s'explique par le fait que la clause vous voulez tout savoir attribuée à un locuteur/énonciateur distinct, est comprise comme étant associée au même locuteur responsable de Elle est malade :

(9) a. Elle est malade puisque vous voulez tout savoir.

b. ? Elle est malade car vous voulez tout savoir.

(ii) Considérons les exemples (10) et (11) :

(10) Puisque vous voulez tout savoir, Paul est communiste.

(11) Juliette s'est absentée, car son fils pourrait avoir mal au genou.

Loin d'être un point de rapprochement entre la clause subordonnée en (10) et la clause introduite par car en (11), la 'justification' que portent celles-ci, les distingue. Il s'agit en fait de deux types de justification relevant, chacun, d'un niveau donné de la structure de la clause. La première justification (e.g. (11)) que nous désignons, après Lyons (1977) et Hengeveld (1996), par la fonction sémantique de Raison se rapporte au niveau propositionnel $\left(\mathrm{X}_{\mathrm{i}}\right)$ puisqu'elle modifie un fait possible. La deuxième justification, elle, que nous convenons de désigner par la fonction de Motivation opère au niveau illocutionnaire : en $\left(10^{\prime}\right)$ le satellite adverbial $\left(\sigma_{4}\right)$ modifie l'acte de langage $\left(\mathrm{E}_{\mathrm{i}}\right)$ comme en témoignent les paraphrases suivantes (où Poss $=$ Possible):

$\left(10^{\prime}\right)=$ Puisque vous voulez tout savoir je vous dis que Paul est communiste.

$=$ La motivation de mon dire est votre désir de tout savoir.

Sur cette base, la différence entre les constructions (10) et (11) peut être représentée comme en (12) et (13) respectivement, et la différence entre les constructions-Motivation et les constructions-Raison peut être représentée comme en (14) :

(12) $\left[D E C L E_{i}:\left[X_{\mathrm{i}}:(\right.\right.$ Paul est communiste) $\left.],\left[D_{\text {ECL E }}:\left[\mathrm{X}_{\mathrm{j}} \text { : (vous voulez tout savoir) }\right]\right]_{\text {Mot }}\right]$

(13) $\left[D E C L E_{\mathrm{i}}:\left[\mathrm{X}_{\mathrm{i}}: \text { (Juliette s'est absentée)], [Poss } \mathrm{X}_{\mathrm{j}} \text { : (son fils avoir mal au genou) }\right]_{\text {Raison }}\right]$

$$
\begin{array}{lll}
(14) & \left(\pi_{4} E_{i}\right)_{\text {Motivation }} & \text { Clause } \\
& \left(\pi_{3} \mathrm{X}_{\mathrm{i}}\right)_{\text {Raison }} & \text { Proposition }
\end{array}
$$

Une éventuelle présence de eh bien ou de puisque dans un texte narratif (cf. les textes de Balzac) traduit une intervention de l'auteur qui joue dans ce cas le même rôle que le locuteur vis-à-vis de son allocutaire dans un échange conversationnel. 
Par ailleurs, les études (e.g. Moeschler et de Spengler 1981, Roulet et al. 1985) effectuées sur mais, quand même, bien que, pourtant, cependant et néanmoins ont montré que ces connecteurs 'contreargumentatifs' constituent trois sous-classes: mais et quand même, bien que et pourtant, cependant et néanmoins. Les connecteurs de la première sous-classe sont considérés comme étant les plus argumentatifs, ceux de la seconde sont conçus comme étant les plus logiques et ceux de la troisième sont envisagés comme constituant une catégorie intermédiaire.

La fréquence d'occurrence d'un seul connecteur (i.e. mais, pourtant et cependant) de chacune des trois sous-classes susmentionnées, dans les trois types de discours examinés (cf. Tableau 5), peut s'expliquer, entre autres, par la parfaite similitude des propriétés que manifeste chaque paire de marqueurs.

L'étude d'un corpus constitué d'exemples tirés d'ouvrages de la littérature française, nous a permis de constater que l'argumentatif mais ne peut mettre en opposition que des propositions relevant de la troisième strate du modèle hiérarchique de la clause prévu en GF (cf. Jadir 2005a). En d'autres termes, lorsqu'un lecteur cherche à découvrir (i.e. interpréter) la fonction argumentative de mais dans un Récit objectif, il procédera à une 'subjectivisation' de celui-ci. Ducrot (1980b : 19) parle dans ce sens d'une intériorisation de la description". En témoigne l'opposition exprimée en (15) dont la compréhension nécessite 'une intériorisation du mouvement de Jeanne en sentiment', i.e. le concevoir non pas en tant que déplacement dans l'espace mais en tant qu'espoir ; l'espoir que la pluie allait s'arrêter. Aussi, le mais de Maupassant est à comprendre comme articulant les deux propositions suivantes: « Jeanne espérait que la pluie cessait, mais la pluie ne cessait pas » :

(15) Jeanne, ayant fini ses malles, s'approcha de la fenêtre, mais la pluie ne cessait pas. (Ducrot 1980b)

Les membres articulés au moyen de mais, qu'il s'agisse du niveau local ou global, font partie d'un texteDiscours où le locuteur/auteur se fixe pour objectif d'arguer pour une cause/conclusion vers laquelle il tente d'incliner son allocutaire/lecteur. Techniquement parlant, le texte où figure la particule connective mais a comme opérateur textuel D arg (Discours argumentatif). Aussi peut-on dire que l'occurrence de mais est co-déterminée par le type de discours.

Corollairement, l'occurrence du connecteur mais dans un texte-Récit amène le lecteur à effectuer un changement de type de discours. Nous ne voulons pour preuve que l'exemple (15) où l'on assiste à une intériorisation d'un geste en mouvement. Ce passage du Récit (narratif ou descriptif) au Discours (argumentatif) au terme d'un processus interprétatif peut être schématisé comme en (16) :

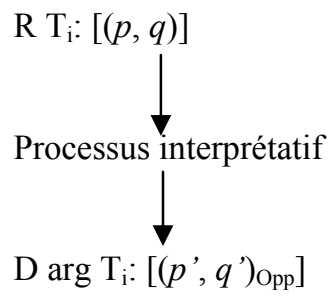

où $p^{\prime}$ 'et $q$ ' renvoient respectivement au résultat de l'interprétation subjective de $p$ et $q$, et Opp indique la fonction sémantique d'Opposition assignée à l'ensemble $p^{\prime}-q$ ' et qui déclenche la réalisation de mais.

Par ailleurs, si Crevels (1998) argue en faveur du fait qu'en espagnol, toutes les strates sémantiques prévues en GF sont pertinentes pour l'expression des clauses concessives, nous avons constaté (cf. Jadir 2000a), qu'en français, la Concession est exprimée au niveau propositionnel, entre autres, au moyen de cependant et pourtant. Ces marqueurs de discours peuvent fonctionner comme des moyens qui effectuent un changement de type de discours. Si le passage d'un type de texte, au moyen de cependant, à un autre semble se rapporter fréquemment au niveau global, il est plutôt plus récurrent dans le cas de pourtant au niveau local. Les données suggèrent également que la fonction de cependant dans ce sens se restreigne à assurer le transfert d'un texte-Discours à un texte-Récit ou, du moins, d'un discours dialogal à un discours monologal. Pour ce qui est de pourtant, en revanche, le transfert peut s'effectuer dans les deux sens, i.e. du Discours au Récit et du Récit au Discours. 
Pour ce qui est de la haute fréquence des trois particules connectives (mais, pourtant et cependant) dans les textes-Récit, elle est due, paraît-il, aux possibilités qu'elles offrent à l'auteur, à des degrés divers, et ce pour préserver ou rompre la maintenance de la continuité du discours (au sens de Givón (1983 et 1989)), i.e. pour assurer la cohérence textuelle. Pour ce qui est des propriétés de changement de types de discours et de maintenance de continuité du discours, considérons les exemples suivants :

(17) a. Il l'avait empoignée, il ne la lâchait pas. Elle s'abandonnait, étourdie par le léger vertige qui lui venait du tas de linge, sans dégoût pour l'haleine vineuse de Coupeau. Et le gros baiser qu'ils échangèrent à pleine bouche, au milieu des saletés du métier, était comme une première chute, dans le lent avachissement de leur vie.

Cependant, madame Bijard nouait le linge en paquets... (ASS: 138-9).

b. Clémence, après avoir plié le dos de la chemise et donné un coup de fer des deux côtés, en était aux poignets et au col. Mais, comme il se poussait toujours contre elle, il lui fit faire un faux pli...

- Madame! dit-elle, empêchez-le donc d'être comme ça après moi!

- Laisse-la, tu n'es pas raisonnable, déclara tranquillement Gervaise. Nous sommes pressées, entends-tu !

Elles étaient pressées, eh bien! quoi? ce n'était pas sa faute. Il ne faisait rien de mal. Il ne touchait pas, il regardait seulement. Est-ce qu'il n'était plus permis de regarder les belles choses que le bon Dieu a faites? Elle avait tout de même de sacrés ailerons, cette dessalée de Clémence! Elle pouvait se montrer pour deux sous et laisser tâter, personne ne regretterait son argent. L'ouvrière, cependant, ne se défendait plus, riait de ces compliments tout crus d'homme en ribote... (ASS:140-141).

Les fragments (17a-b) montrent que cependant est un marqueur de changement de type de discours; l'auteur s'en sert pour passer d'un texte-Discours à un texte-Récit, i.e. pour reprendre une continuité discursive interrompue au moyen d'un passage modalisé subjectivement. La reprise de la chaîne narrative en (17a) moyennant cependant s'effectue après un Discours commentatif dans lequel l'auteur prédit l'avenir du couple conjugal. Qui plus est, le passage produisant la rupture de la continuité du Récit peut être un discours indirect libre comme en témoigne l'exemple (17b). Le segment introduit par la particule cependant brise le discours subjectif du personnage pour lui succéder celui du narrateur ${ }^{10}$.

Dans le texte (17a), le marqueur cependant fonctionne au niveau global; la reprise de la narration coïncide avec la reprise d'un Topique déchu (e.g. madame Bijard) qui ne coréfère pas, toutefois, avec le Topique qui précède directement le texte-Discours. En revanche, le connecteur en question en (17b) est employé au niveau local; le Topique Repris (i.e. l'ouvrière) contenu dans le Récit récupéré au moyen de cependant est coréférentiel avec le Topique Donné (i.e. Clémence) de la séquence interrompue, antéposée au discours marqué subjectivement.

Il s'ensuit que cependant est de surcroît un marqueur de reprise d'un type de discours déchu. Lors de cette stratégie de maintenance discursive, si la particule connective cependant est employée globalement, elle se caractérise par son pouvoir de reprendre un élément Topique se situant après une longue distance référentielle (e.g. (17a)), alors qu'employée localement, elle tend à récupérer préférentiellement le Topique de la chaîne récemment brisée (e.g. (17b)).

Pour ce qui est de la fonction de pourtant en tant que marqueur de changement de type de discours, considérons les fragments suivants :

$$
\text { a. - Qu'est- ce que tu fais ?... Où vas-tu ? }
$$

Elle ne répondit pas d'abord. Puis, lorsqu'il répéta sa question, furieusement, elle se décida.

- Tu le vois bien, peut-être... Je vais laver tout ça... [...] puis il jeta le tout [les loques] sur les bras de Gervaise en disant: 
- Tiens, porte ça au clou.

- Tu ne veux pas que je porte aussi les enfants? demanda-t-elle.

Hein! si l'on prêtait sur les enfants, ce serait un fameux débarras!

Elle alla au Mont-de-Piété, pourtant... (ASS: 19).

b. La noce se retourna sur ses pas, traversa de nouveau le salon carré et la galerie d'Apollon... Mais le cartonnier voulait montrer à Lorilleux les bijoux anciens. Ça se trouvait à côté, au fond d'une petite pièce, où il serait allé les yeux fermés. Pourtant, il se trompa, égara la noce le long de sept ou huit salles, désertes, froides... (ASS: 78).

En dépit de sa grande tendance à fonctionner au niveau local, les données suggèrent que le connecteur pourtant permette de reprendre la continuité rompue d'un texte narratif aussi bien au niveau global qu'au niveau local, comme le montrent les segments (18a) et (18b). Dans ces exemples, l'apparition de pourtant déclenche le retour de la narration après un Discours conversationnel et un discours indirect libre respectivement. La haute fréquence de la coïncidence de pourtant avec un Topique faible (i.e. un pronom personnel) est confirmée par ces deux passages; le recours à ce type d'anaphores s'explique par la courte distance entre les Topiques Donnés coréférents, ce qui ne risque pas de créer d'ambiguïté référentielle nécessitant l'emploi d'un codage matériel fort.

Normalement les connecteurs du type de tandis que qui produisent une rupture de la continuité topicale au moyen d'un parallélisme contrastif sont plus fréquents dans les correspondances qui se distinguent par la diversité de leurs thèmes que dans les narrations qui sont marquées par leur haute continuité textuelle. La fréquence très basse de tandis que dans les $\mathrm{LD}$, comme le suggèrent les données, s'est effectuée au profit de la grande récurrence d'autres moyens assumant la même fonction discursive tels que quant $\grave{a}$, alors que...

En termes de GF, tandis que introduit, au niveau prédicationnel, un satellite $\sigma_{2}$ affecté de la fonction sémantique de Circonstance (Cir); au niveau du discours, la particule marque un satellite textuel $\sigma_{2}$ qui spécifie la Circonstance des séquences principales de la narration. En témoigne l'exemple (19) tiré de L'Assommoir, p. 42 :

(19) La fruitière retirait sa dernière poêlée de pommes de terre frites, tandis que le charcutier remettait en ordre des assiettes débandées de son comptoir... (ASS: 42).

La prédication enchâssée par tandis que ne fournit pas de spécification précise sur le temps du commencement ou de l'achèvement de l'EdC exprimé par la prédication matricielle. Elle marque, au contraire, une simultanéité entre les deux EdC en présence. En d'autres termes, la construction subordonnée tandis que le charcutier remettait...spécifie une circonstance de la construction principale la fruitière retirait...

Sur cette base, l'assignation de la fonction sémantique de Cir(constance) au niveau de la strate prédicationnelle peut être représentée de la manière suivante:

$$
\left(\operatorname{Sim} \pi_{2} \mathrm{e}_{\mathrm{i}}\right)_{\mathrm{Cir}} \quad \text { Prédication }
$$

où Sim est un opérateur indiquant la simultanéité.

De même, la fonction de Focus Parallèle (Foc Par) marquée par le moyen de focalisation tandis que peut être assignée à un seul élément ou à la totalité de la phrase quand le contraste est établi entre tous les membres d'une construction parallèle (i.e. la fonction de Listing selon Geluykens 1992). En (21) le parallélisme syntaxique implique plus d'une seule paire d'éléments opposés relevant de catégories grammaticales différentes. Le contraste est établi entre deux SN un morceau de fromage blanc/de grosse fraises meurtries, deux adverbes à gauche/à droite, deux verbes nageait/s'entassaient et deux syntagmes prépositionnels dans un plat creux/dans un autre plat : 

autre plat, à droite, s'entassaient de grosses fraises meurtries dont le jus coulait (ASS: 208).

L'assignation de la fonction pragmatique de Foc Par, au plan textuel, à toute une prédication peut être schématisée comme en (22) :

$$
\text { R T } \left.\left.\left.\left.\left.T_{\mathrm{i}} \text { : [DECL ([e } \mathrm{e}_{\mathrm{i}} \text { : Prédication }\left(\mathrm{e}_{\mathrm{i}}\right)\right] \text {, [ } \mathrm{e}_{\mathrm{j}} \text { : Prédication }\left(\mathrm{e}_{\mathrm{j}}\right)\right]\right)_{\text {Foc Par }}\right]\right]
$$

Enfin, le connecteur français tandis que est un moyen pertinent à la création de la cohérence en ce sens qu'il assure l'introduction d'un Topique Nouveau ou d'un Sous-Topique ou le changement d'un Topique (d'une action ou d'un 'setting'), ce qui produit une discontinuité discursive (cf. Jadir 2000b).

L'examen des données permettrait, d'autre part, de conclure que le causal car est d'une haute fréquence dans le Discours conversationnel et dans les lettres (contrairement au Récit), alors que parce que est d'une fréquence supérieure dans les Récits comparativement aux conversations et aux correspondances. Ce constat pourrait, a priori, être expliqué sur la base de l'hypothèse stipulant que le connecteur car implique deux actes d'énonciation (deux 'actes de discours' en termes de Hengeveld et Mackenzie 2008 : 54), tandis que le connecteur parce que suppose un acte d'énonciation unique. En termes de GF, parce que introduit des prédications exprimant des $\mathrm{EdC}$, i.e. des entités du second ordre, car, quant à lui, marque des propositions désignant des faits possibles, i.e. des entités du troisième ordre. Aussi, convenons-nous d'assigner la fonction sémantique de Cause à la clause adverbiale enchâssée au moyen de parce que, et celle de Raison à la construction introduite par car (voir supra). Considérons les exemples (23) et (11) repris ici pour convenance :

(23) La voiture a glissé parce que la route était mouillée.

(11) Juliette s'est absentée, car son fils aurait mal au genou.

En (23) la construction enchâssée décrit l'événement motivant, i.e. causant l'événement de la construction enchâssante, et il est clair qu'aucun participant à l'EdC n'est impliqué dans cette motivation. En (11) la clause introduite par car ne constitue pas une cause réelle de l'événement/l'EdC exprimé dans la clause précédente, mais elle désigne plutôt l'idée ou, en termes de Hengeveld, 'le contenu propositionnel' qui mène le contrôleur de l'EdC (e.g. Juliette) à s'engager dans cet EdC.

Sur cette base, la différence entre les constructions (23) et (11) peut être représentée comme en (24) et (25) respectivement et la différence entre les constructions-Cause et les constuctions-Raison peut être représentée comme en (26) (où l'opérateur 'Poss' = Possible) :

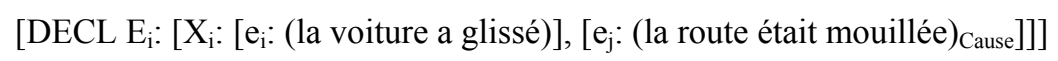

[DECL E $\mathrm{i}_{\mathrm{i}}$ : [ $\mathrm{X}_{\mathrm{i}}$ : (Juliette s'est absentée)], [Poss $\mathrm{X}_{\mathrm{j}}$ : (son fils avoir mal au genou) $]_{\text {Raison }}$ ]

$$
\begin{array}{ll}
\left(\pi_{2} \mathrm{e}_{2}\right)_{\text {Cause }} & \text { Prédication } \\
\left(\pi_{3} \mathrm{X}_{\mathrm{i}}\right)_{\text {Raison }} & \text { Proposition }
\end{array}
$$

En revanche, tout porte à croire que le résultat auquel nous avons abouti ne semble pas tout à fait complet. Effectivement, quelques données, attestées, suggèrent que le marqueur de cause parce que manifeste un comportement double: en plus de l'enchâssement d'une prédication (e.g. (23)) il peut assurer celui d'une proposition (e.g. (28a-c)); ce second emploi le rapproche de car qui introduit souvent une structure propositionnelle (e.g. (27 a-b)):

(27) a. A présent, ma chère amie, si je suis bien aise, je suis aussi bien embarrassée, car sûrement il ne faut pas que je réponde à cette lettre-là (LD: 56).

b. Elle était touchée, elle trouvait Coupeau très honnête; car elle avait bien cru un moment que c'était fini, qu'il allait coucher là (ASS: 53).

(28) a. J'espère aussi que vous ne direz à personne que je vous ai écrit; parce que sûrement on m'en blâmerait, et que cela pourrait me causer bien du chagrin (L D: 61). 
b. Madame Putois offrit de se retirer, parce que, selon elle, il ne fallait pas jouer avec ça (ASS: 198).

c. Mademoiselle Remanjou fermait les yeux, parce qu'elle croyait marcher sur l'eau (ASS: 76).

Il découle des exemples ci-dessus que parce que, à l'instar de car, peut introduire une proposition comme le montre la possibilité de co-occurrence des deux marqueurs avec des procédés linguistiques identiques. En (28c), comme en (27b), la proposition comporte un prédicat d'attitude intellectuelle (Dik 1997b) (i.e. croire) qui spécifie l'attitude d'une personne en relation avec le fait possible désigné par le complément propositionnel. En (28a-b), comme en (27a), la proposition est marquée par des satellites du niveau 3 comme l'Attitude (Att) sûrement et la Source (So) selon elle qui modalisent subjectivement le contenu propositionnel.

Si cette remarque est tenable, nous pouvons supposer que, parallèlement au because anglais, le parce que peut avoir deux emplois relevant de deux strates distinctes de la clause (la prédication et la proposition), et ce en fonction du type d'entité auquel on réfère: un Etat de Choses (e) ou un fait possible (X).

Somme toute, alors que l'emploi de car présuppose un texte-Discours assurant l'intervention du locuteur, le recours à parce que présuppose un texte-Récit dans lequel la clause adverbiale présente les circonstances de la clause principale.

Dans le même ordre d'idées, un auteur/locuteur se sert des connecteurs causaux du type de même, d'ailleurs et en effet en vue d'appuyer un point de vue par l'adjonction d'un certain nombre d'arguments supplémentaires. De là, on ne s'attendait pas à rencontrer ce genre de particules dans un Récit puisqu'elles supposent un locuteur/auteur et un allocutaire/lecteur (réel ou potentiel) et chez le premier l'intention de convaincre l'autre de la vérité d'une cause en faveur de laquelle il argue. Aussi peut-on avancer que ces connecteurs sont candidats à apparaitre fréquemment dans un type de discours précis, le Discours argumentatif.

Concernant les marqueurs de consécution, nous avons remarqué que le Récit narratif opte préférentiellement pour le connecteur alors, tandis que le Discours conversationnel recourt excessivement à donc. Un tel constat trouverait son explication dans la disparité des propriétés que révèle chacun des deux connecteurs.

En effet, alors que alors instaure toujours un lien consécutif entre les propos d'un locuteur et la conséquence que l'on en retire, donc est le seul à pouvoir introduire un 'acte directeur' qui est la reprise d'un acte déjà énoncé sans impliquer de lien consécutif (e.g. (29a-b)). En d'autres termes, à l'encontre de alors, donc permet de récupérer une action et un Topique (Top Rep) sans marquer de consécution. Bien plus, si l'emploi des connecteurs alors, donc et par conséquent entraîne une prise en charge du locuteur d'un 'acte directeur', alors se distingue par la possibilité de ne pas assumer l'acte performé (e.g. (30a-b)). Cette neutralité correspond au trait d'objectivité qui caractérise les textes-Récit. Une dernière caractéristique de alors réside dans le fait que, contrairement à donc, il ne fait pas intervenir de norme (idéologique ou culturelle) qui légitime le processus déductif (e.g. (31a-b)). En termes de GF, l'interprétation des occurrences d'emploi de donc l'emporte sur celles de alors quant au recours au Modèle de l'Utilisateur du Langage Naturel (MULN), i.e. tandis que alors se limite au module grammatical, donc nécessite l'interaction de tous les modules pour une meilleure interprétation de ses usages, principalement, le module grammatical, le module épistémique et le module logique. En témoignent les exemples ci-dessous empruntés à (Roulet et al. 1985) :

(29) a- Ce soir le thème de cette émission [...]

$$
\begin{aligned}
& \text { nous parlerons tout d'abord du rôle }[\ldots] \\
& \text { puis nous parlerons de la politique }[\ldots] \\
& \text { et enfin de la défense nationale }[\ldots]
\end{aligned}
$$

donc premier thème le problème du rôle $[\ldots]$ 
b- $(*$ alors $)$ premier thème le problème du rôle $[\ldots]$

(30)

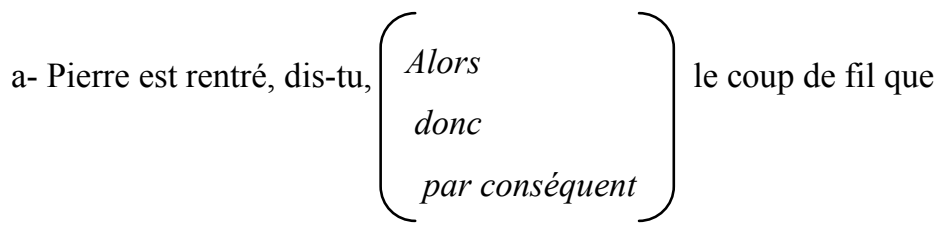

nous venons de recevoir, c'est une farce.

b- Pierre serait rentré, dis-tu, $\left(\begin{array}{l}\text { Alors } \\ * \text { donc } \\ * \text { par conséquent }\end{array}\right)$ le coup de fil que nous venons de recevoir

de l'étranger, c'est une farce?

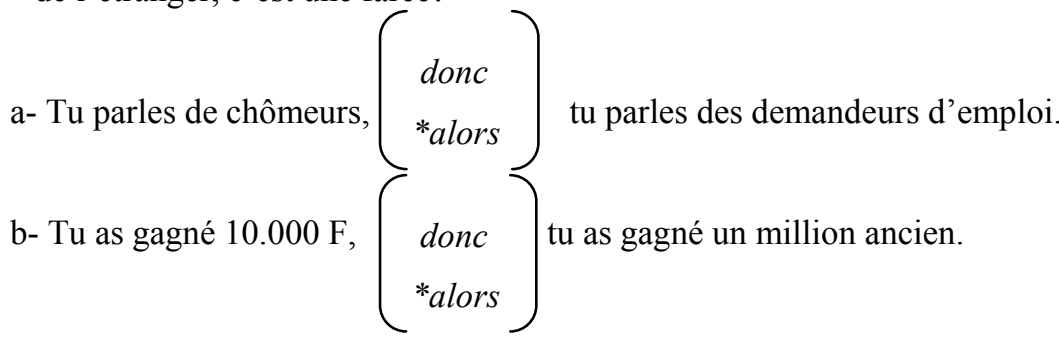

\section{Conclusion}

Dans cet article, nous avons examiné l'impact du type de discours dans la codétermination du type de marqueurs appropriés. Aussi, la comparaison établie entre la dichotomie benvenistienne Récit/Discours et la dichotomie genevoise monlogal/dilogal a-t-elle montré que le type de discours impose ses restrictions dans la sélection de plusieurs propriétés linguistiques (e.g. formes verbales et personnelles, deixis, verbes modaux et performatifs). Au reste, l'adoption du modèle hiérarchique et modulaire de la clause (i.e. la Grammaire Fonctionnelle) nous a permis d'expliquer fonctionnellement les résultats des statistiques que nous avons effectuées de la fréquence des différents types de particules connectives (e.g. causales, adversatives, consécutives) relevées dans un corpus diversifié (théâtre, roman, roman épistolaire) de la littérature française.

\section{Références bibliographiques}

Bakhtine, M. (1970). La poétique de Dostö̈evski. Seuil: Paris.

Bakhtine, M. (1978). Esthétique et théorie du roman. Gallimard: Paris.

Benveniste, E. (1966). Problèmes de linguistique générale. Seuil: Paris.

Bolkestein, A. M. 1998. What to do with Topic and Focus ? Evaluating pragmatic information. In Hannay, M. \& M. Bolkestein (eds.), Functional Grammar and Verbal Interaction. J. Benjamins: Amsterdam/Philadelphia, 193-214.

Bolkestein, A. M. 2000. Discourse organization and anaphora in Latin. In Herring, C. et al. (eds.), Textual Parameters in Older Languages. John Benjamins: Amsterdam/ Philadephia, 107-137.

Brown, G. \& G. Yule. (1983). Discourse analysis. Cambridge University Press: Cambridge.

Crevels, M. (1998). Concession in Spanish. In Hannay, M. \& M. Bolkestein (eds.), Functional Grammar and Verbal Interaction. J. Benjamins : Amsterdam/Philadelphia, 129-148.

Dik, S.C. (1997a). The theory of Functional Grammar. Part 1: The structure of the clause. (Edited by Kees Hengeveld). Mouton de Gruyter: Berlin/New York. 
Dik, S.C. 1997b. The theory of Functional Grammar. Part 2 : Complex and Derived Constructions. (Edited by Kees Hengeveld). Berlin/New York : Mouton de Gruyter.

Ducrot, O. et al. (1980a). mais occupe-toi d'amélie. In Ducrot et al. (eds.), Les mots du discours. Ed. Minuit: Paris, 93-130.

Ducrot, O. (1980b.). Analyses pragmatiques. Communications 32, 11-60.

Ducrot, O. (1982). Note sur l'argumentation et l'acte d'argumenter. Cahiers de Linguistique française 4, 143-163.

Ducrot, O. (1983). Opérateurs argumentatifs et visée argumentative. Cahiers de Linguistique française 5, 7-36.

Ducrot, O. (1984). Le dire et le dit. Minuit : Paris.

Geluykens, R. (1992). From discourse process to grammatical construction. On left-dislocation in English. Amsterdam/Philadelphia : J. Benjamins.

Givón, T. (1983). Topic continuity in discourse : an introduction. In Givón, T. (ed.), Topic continuity in discourse : a quantitative cross-linguistic study. J. Benjamins: Amsterdam/Philadelphia, 5-41.

Givón, T. (1989). Mind, Code and Context : Essays in Pragmatics. Lawrence Erlbaum Hillsdale: New Jersy.

Hansen, M-B. M. (1996a). Eh bien : marker of comparison and contrast. In Engberg- Pedersen, E. et al. (eds.), Content, expression, and structure. Studies in Danish Functional Grammar. J. Benjamins: Amsterdam/Philadelphia, 315-342.

Hansen, M-B. M. (1996b). The function of discourse particles. A study with special reference to spoken standard French. Dissertation. University of Copenhagen.

Hengeveld, K. (2004a). The Architecture of a Functional Discourse Grammar. In Mackenzie, J.-L. \& Gómez González (eds.), A new architecture for Functional Grammar. Mouton de Gruyter: Berlin/New York, 1-21.

Hengeved, K. (2004b). Epilogue. In Mackenzie, J.-L \& Gómez González (eds.), A new architecture for Functional Grammar. Mouton de Gruyter: Berlin/New York, 365-378.

Hengeveld, K. \& J.L. Mackenzie. (2006). Functional Discourse Grammar. In K. Brown (ed.), Encyclopedia of Language and Linguistics ( $2^{\mathrm{e}}$ édition) Vol. IV. Oxford: Elsevier, 668-676.

Hengeveld, K. \& J.L. Mackenzie. (2008). Functional Discourse Grammar. Oxford University Press: Oxford.

Hymes, O. (1972). On communicative competence. In Pride, J. B. \& J. Holmes (eds.), Sociolinguistics. Penguin: Hardmonds, 269-293.

Jadir, M. (1995). Focus et progression textuelle. In Approches du récit. Publications de la Faculté des Lettres et des Sciences Humaines de Mohammedia. Najah El Jadida: Casablanca, 93-115.

Jadir, M. (1998). Textual cohesion and the notion of perception. In Hannay, M. \& M. Bolkestein (eds.), Functional Grammar and Verbal Interaction. J. Benjamins: Amsterdam/Philadelphia, 43-58.

Jadir, M. (2000a). Types de discours, marqueurs de discours et cohésion textuelle : le cas de pourtant et cependant. In Bustos J. J. \& P. Charaudeau, J. L. Girón \& S. Iglesias \& G. López (eds.), Lengua. Discurso, Texto (I Simposio Internacional de Analisis del Discurso). Visor Libros: Madrid, 591-601.

Jadir, M. (2000b). Discourse coherence : a case study of the French connector tandis que. Hermes Journal of Linguistics 25, 93-112.

Jadir, M. (2005a). La cohérence du discours en Grammaire Fonctionnelle: le cas du texte narratif (Préface de Machtelt Bolkestein). Ed. Bouregreg: Rabat.

Jadir, M. (2005b). Marqueurs de discours et cohérence du discours : le cas de car, parce que et puisque. In Hermes Journal of Linguistics 34, 169-197.

Jadir, M. (2006). Grammaire Fonctionnelle et paramètre textuels (Préface de Co Vet). Ed. Bouregreg: Rabat.

Jadir, M. (2009). Grammaire Fonctionnelle (du Discours): Evaluation et perspectives. In Hermes Journal of Language and Communication Studies 43, 163-201.

Kroon, C. (1994). Discourse connectives and discourse type : the case of Latin at. In Herman, J. (ed.), Linguistic studies on Latin. J. Benjamins: Amsterdam / Philadelphia, 303-317. 
Kroon, C. (1995). Discourse particles in Latin : a study of nam, enim, autem, vero and at. Gieben: Amsterdam.

Kroon, C. (1997). Discourse markers, discourse structure and Functional Grammar. In Connolly et al. (eds.), Discourse and Pragmatics in Functional Grammar. Mouton de Gruyter: Berlin/New York, 17-32.

Levelt, W. J. M. (1989). Speaking : From Intention to Articulation. MA : MIT Press.

Longacre, R. (1983). The grammar of discourse. Plenum: New York.

Moeschler, J. (1996). Théorie pragmatique et pragmatique conversationnelle. Armand Colin: Paris.

Moeschler, J. \& N. de Spengler. (1981). Quand même: de la concession à la réfutation. Cahiers de Linguistique française 2, 93-112.

Moutaouakil, A. (1998). Benvenist's 'Récit' and 'Discours' as discourse operators in Functional Grammar. In Hannay, M. \& M. Bolkestein (eds.), Functional Grammar and Verbal Interaction. J. Benjamins: Amsterdam/Philadelphia, 25-41.

Reboul, A. \& J. Moeschler. (1998). Pragmatique du discours. De l'interprétation de l'énoncé à l'interprétation du discours. Armand Colin: Paris.

Risselada, R. (1998). The discourse functions of sane: Latin marker of agreement in description, interaction and concession. Journal of Pragmatics 30, 225-244.

Roulet, E. et al. (eds.). (1985). L'articulation du discours en français contemporain. Lang: Berne.

Schiffrin, D. (1987). Discourse markers. Cambridge: University Press Cambridge.

Sirdar-Iskandar, Ch. (1980). Eh bien! Le Russe lui a donné cent francs. In Ducrot et al. (eds.), Les mots du discours. Ed. Minuit: Paris, 161-191.

Stubbs, M. (1983). Discourse analysis. Basil Blackwell: Oxford.

Vet, C. (1998). The multilayered structure of the utterance : about illocution, modality and discourse moves. In Hannay, M. \& M. Bolkestein (eds.), Functional Grammar and Verbal Interaction. J. Benjamins: Amsterdam/Philadelphia, 1-23.

Vries, L. de. (1989). Studies in Wambon and Kobai : aspects of two Papuan languages of Irian Jaya. [Ph. D. dissertation, University of Amsterdam.]

\footnotetext{
${ }^{1}$ La GF prévoit deux types de fonctions pragmatiques : les fonctions pragmatiques externes (e.g. Thème, Queue, etc.) et les fonctions pragmatiques internes (i.e. Topique et Focus). Le Topique, dit 'Topique de Discours' (Top Disc) puisqu'il caractérise les entités à propos desquelles l'information est fournie dans le discours, subsume plusieurs sous-fonctions : le Topique Nouveau (Top Nouv), le Topique Donné (Top Don), le Sous-Topique (Sous Top) et le Topique Repris (Top Rep). Le Focus, qui caractérise l'information la plus saillante, englobe les deux types principaux suivants : le Focus de Nouveau (Foc Nouv) ou Focus Complétif (Foc Compl) et le Focus de Contraste qui comprend le Focus Parallèle (Foc Par) et le Focus Contre-présuppositionnel.

${ }^{2}$ La prédication nucléaire contient un prédicat (Pred) et un certain nombre d'arguments $\left(\arg ^{\mathrm{n}}\right)$. Cette prédication est convertie en une prédication centrale par l'association d'un opérateur de prédicat $\left(\pi_{1}\right)$ qui désigne 'les distinctions aspectuelles' et d'un satellite de prédication $\left(\sigma_{1}\right)$ qui désigne la Manière, la Vitesse et l'Instrument. La prédication centrale est étendue par l'adjonction d'un opérateur de prédication $\left(\pi_{2}\right)$ qui concerne les traits temporels, les traits modaux et la polarité ('positif' (Pos) vs 'Négatif' (Neg)) et d'un satellite $\left(\sigma_{2}\right)$ prédicationnel qui renvoie, e.g. au Temps, au Lieu, au But, à la Cause, etc. La conversion de cette prédication étendue $\left(\mathrm{e}_{\mathrm{i}}\right)$ qui exprime un Etat de Choses $(\mathrm{EdC})$ en une proposition $\left(\mathrm{X}_{\mathrm{i}}\right)$ qui exprime un fait possible (FP) s'effectue par l'adjonction d'un opérateur propositionnel $\left(\pi_{3}\right)$ et, éventuellement, d'un satellite de niveau $3\left(\sigma_{3}\right)$ qui spécifient l'évaluation ou l'attitude du Locuteur $(\mathrm{L})$ vis-à-vis du contenu propositionnel. L'incorporation du niveau de la proposition dans le niveau supérieur, i.e. le niveau de la clause, qui désigne l'acte de discours (noté Ei), peut être marquée par un opérateur illocutionnaire $\left(\pi_{4}\right)$ indiquant le type basique de la clause (i.e. DECL(aration), INT(errogation),
} 
IMP(ératif) et EXCL(amation)) et, éventuellement, un satellite illocutionnaire $\left(\sigma_{4}\right)$ qui spécifie la manière dont $\mathrm{L}$ désire que l'acte soit compris par l'Allocutaire (A). La forme générale de la clause peut ainsi être représentée de la manière suivante :

(i) $\left[\pi_{4} \mathrm{E}_{\mathrm{i}}:\left[\pi_{3} \mathrm{X}_{\mathrm{i}}:\left[\pi_{2} \mathrm{e}_{\mathrm{i}}:\left[\pi_{1:}:\left[\operatorname{Pred}_{\arg ^{\mathrm{n}}}^{\mathrm{n}}\right]\left(\sigma_{1}\right)\right]\left(\sigma_{2}\right)\right]\left(\sigma_{3}\right)\right]\left(\sigma_{4}\right)\right]$

${ }^{3}$ Une nouvelle version de la Grammaire Fonctionnelle (la Grammaire Fonctionnelle du Discours (GFD)), présentée dans (Hengeveld 2004a,b) et (Hengeveld et Mackenzie 2006 et 2008), constitue une tentative de combiner les deux approches; elle se veut hiérarchique et modulaire. Elle s'inspire des recherches psychologiques (cf. Levelt 1989) et fonctionne, de ce fait, de façon 'haut-bas' (top-down), puisqu'elle rend compte de la structure des expressions linguistiques en tant que résultat de la décision du Locuteur à travers un processus de production qui s'étend de l'intention vers l'expression via la formulation, l'encodage et l'articulation. Pour une évaluation du modèle de Dik et celui de Hengeveld \& Mackenzie, voir Jadir (2009).

${ }^{4}$ Quoique le texte (3) soit moins marqué que les textes (4) et (5) et que les textes narratifs du type de (3) constituent l'écrasante majorité dans la littérature française classique, certains textes témoignent de la possibilité de la coexistence de la forme personnelle (je/tu) avec le passé simple (e.g. Carmen de P. Mérimée) et de la non-personne (il/elle) avec le passé composé (e.g. L'Etranger d'A. Camus).

${ }^{5}$ Dans la littérature de l'Ecole de Genève (cf. Roulet et al. 1985), le locuteur est celui qui produit le discours dans toutes ses formes de réalisation (e.g. monologal, dialogal). Le scripteur est l'équivalent du locuteur quand il s'agit d'une production écrite. L'énonciateur désigne celui qui prend en charge une intervention. L'enchâssement des interventions les unes dans les autres permet de distinguer l'énonciateur principal, qui prend en charge une intervention constitutive d'échange, d'énonciateurs subordonnés, qui prennent en charge les interventions constitutives d'interventions. Par ailleurs, le terme auteur, au sens de 'voix', semble renvoyer délibérément au locuteur (p. 71), à l'énonciateur (p. 72-3) et au destinateur (p. 73). Ducrot (1984 : 189-192), quant à lui, s'oppose à «l'unicité» du sujet parlant, i.e. au point de vue 'unicitaire' de la linguistique qui attribue à l'énoncé un seul sujet. Il distingue entre «le sujet parlant», l'auteur réel du discours, i.e. l'agent d'un acte sémantique, « le locuteur », i.e. l'agent qui prend en charge l'énonciation impliquée dans l'énoncé et «l'énonciateur »,i.e. la source de points de vue exprimés dans l'énonciation.

${ }^{6}$ Si pour Benveniste (1966) les correspondances relèvent du Discours, plusieurs linguistes (e.g. Longacre 1983, Roulet et al. 1985, Bolkestein 2000) les considèrent comme ne représentant pas un type de texte. Pour Longacre, par exemple, les lettres peuvent contenir un mélange de fragments de discours (narratif, expositif, etc.).

${ }^{7}$ Si l'échantillon qui nous a servi de corpus (3 ouvrages renvoyant chacun à un type de texte) ne permet de se décider sur une éventuelle généralisation des résultats concernant la codétermination du type de marqueurs par le type de texte, il semble que la dizaine de recherches que nous avons dirigées et qui ont traité de la même problématique à partir de corpus diversifiés de la littérature française, est à même de prétendre à la représentativité et semble corroborer les résultats obtenus dans ce travail.

${ }^{8}$ Sirdar - Iskandar (1980 : 161) définit le rôle que remplissent les morphèmes interjectifs du type de eh bien de la manière suivante: «L'énonciateur peut... se présenter comme agissant sur autrui en le faisant entrer dans son jeu, en le forçant en quelque sorte à tirer une conclusion qu'il a lui-même déterminée à l'avance». Cette définition va dans le sens de la définition du Discours de Benveniste (1966). (cf. aussi Hansen 1996a, b).

${ }^{9}$ Notons que Ducrot (1980b : 19) signale, dans le même sens, que mais dispose de deux miracles :

1- il oblige le lecteur à introduire des résonances affectives dans une description qui, prise en elle même, apparaîtrait purement 'objective',

2- l'intériorisation de la description - appelée, paradoxalement, par le fait qu'il s'agit d'une description purement extérieure -, 'voilà le deuxième miracle que j'attribuerais à mais'. 
${ }^{10}$ Pour Barthes (1966: 25) «narrateur et personnages sont essentiellement des 'êtres de papier'; l'auteur (matériel) d'un récit ne peut se confondre avec le narrateur de ce récit». Cette dichotomie est très importante dans la mesure où elle concourt à une meilleure caractérisation de la distinction benvenistienne Discours-Récit. Ainsi, dirons-nous que, dans un texte donné, le Récit historique est le produit de l'entité fictive qui est le narrateur. C'est donc un texte marqué par l'exclusion de toue référence à l'instance discursive qui le produit, (i.e. son auteur « en chair et en os »). Inversement, le Discours regroupe l'ensemble des 'enclaves' discursives renvoyant à la personne de l'auteur. 